\title{
Cost-Effective Fabrication of Transparent Strain Sensors via Micro-Scale 3D Printing and Imprinting
}

\author{
Rui Wang ${ }^{1,2}$, Xiaoyang Zhu ${ }^{1,2, *}$, Luanfa Sun ${ }^{1,2}$, Shuai Shang ${ }^{1,2}$, Hongke Li ${ }^{1,2}$, Wensong Ge ${ }^{1,2}$ \\ and Hongbo Lan 1,2,*
}

1 Key Lab of Industrial Fluid Energy Conservation and Pollution Control, Ministry of Education, Qingdao 266520, China; wr1970627@163.com (R.W.); sunluanfa@163.com (L.S.); shangshuai0602@163.com (S.S.); LHK1164072308@163.com (H.L.); g17864208754@126.com (W.G.)

2 Shandong Engineering Research Center for Additive Manufacturing, Qingdao University of Technology, Qingdao 266520, China

* Correspondence: zhuxiaoyang@qtech.edu.cn (X.Z.); hblan99@126.com (H.L.)

Citation: Wang, R.; Zhu, X.; Sun, L.; Shang, S.; Li, H.; Ge, W.; Lan, H. Cost-Effective Fabrication of Transparent Strain Sensors via Micro-Scale 3D Printing and Imprinting. Nanomaterials 2022, 12, 120. https://doi.org/10.3390/ nano12010120

Academic Editor: Jun Taniguchi

Received: 29 November 2021

Accepted: 28 December 2021

Published: 30 December 2021

Publisher's Note: MDPI stays neutral with regard to jurisdictional claims in published maps and institutional affiliations.

Copyright: (c) 2021 by the authors. Licensee MDPI, Basel, Switzerland. This article is an open access article distributed under the terms and conditions of the Creative Commons Attribution (CC BY) license (https:// creativecommons.org/licenses/by/ $4.0 /)$.

\begin{abstract}
The development of strain sensors with high sensitivity and stretchability is essential for health monitoring, electronic skin, wearable devices, and human-computer interactions. However, sensors that combine high sensitivity and ultra-wide detection generally require complex preparation processes. Here, a novel flexible strain sensor with high sensitivity and transparency was proposed by filling a multiwalled carbon nanotube (MWCNT) solution into polydimethylsiloxane (PDMS) channel films fabricated via an electric field-driven (EFD) 3D printing and molding hybrid process. The fabricated flexible strain sensor with embedded MWCNT networks had superior gauge factors of 90,285 , and 1500 at strains of $6.6 \%, 14 \%$, and $20 \%$, respectively. In addition, the flexible strain sensors with an optical transparency of $84 \%$ offered good stability and durability with no significant change in resistance after 8000 stretch-release cycles. Finally, the fabricated flexible strain sensors with embedded MWCNT networks showed good practical performance and could be attached to the skin to monitor various human movements such as wrist flexion, finger flexion, neck flexion, blinking activity, food swallowing, and facial expression recognition. These are good application strategies for wearable devices and health monitoring.
\end{abstract}

Keywords: multiwalled carbon nanotube; strain sensors; transparent sensors; micro-scale 3D printing

\section{Introduction}

Flexible strain sensors play an important role in applications such as health monitoring [1-3], electronic skin [4-6], wearable devices [7-12], and human-computer interactions [13]. Piezoresistive strain sensors have played an important role in the field of flexible strain sensors due to their simple reading mechanism. In which, Sensitivity and tensile properties are two important parameters for evaluating the performance of sensors $[14,15]$. Parameters such as response time and dynamic stability are also crucial for their practical applications. Hence, the development of flexible strain sensors with high sensitivity and stretchability is crucial for monitoring of human motion. However, most strain sensors are opaque to ensure sensitivity and tensile properties, which limits their practical application in areas such as the face, neck, and other visible areas.

Here, polydimethylsiloxane (PDMS) [16-18], thermoplastic polyurethane (TPU) [19], Ecoflex [20], and natural rubber [21] were selected as base materials for flexible strain sensors. PDMS is optically transparent, flexible, scalable, and can be easily combined with electronic materials; thus, it is a popular material for flexible wearable strain sensors. Different conductive fillers such as metallic nanoparticles [22,23], silver nanowires (AgNWs) [24,25], carbon nanotubes (CNTs) [26-28], and graphene [29,30] have been used to prepare strain sensors. Of these, metal nanoparticles and AgNWs have high conductivity and can obtain high conductivity with low filler amounts. However, metal fillers are 
difficult to process, and AgNWs are rarely used to fabricate flexible strain sensors due to their costs [31,32]. Although graphene has a high carrier mobility and large specific surface area, it is difficult to uniformly disperse it in polymers, which limits the application of graphene in flexible strain sensors [33]. CNTs have a small percolation threshold due to its nanometer scale, high aspect ratio, and excellent electrical conductivity. CNTs have stable properties and maintain their original electrical properties even under tension and bending, making them particularly suitable for the preparation of flexible strain sensors $[34,35]$. The combination of PDMS and CNTs has been used to prepare strain sensors with high sensitivity and stretchability. However, published CNTs are mostly randomly distributed in the polymer matrix, thus resulting in compromised optical transparency of the strain sensors.

Recent research has been devoted to modifying the conductive structure of nanomaterials to enhance their sensor performance, including microcracks, microspheres, and microporosity. Of these, microcrack structures inspired by the spider structure have been widely used to prepare various flexible strain sensors with high sensitivity. For example, Zhou et al. [36] sprayed CNT on TPU and produced cracks by pre-stretching. The prepared CNT/TPU strain sensor has an ultra-wide detection range of up to $300 \%$ with a gauge factor of 428.5 in the strain range of $0-100 \%, 9268.8$ in the strain range of $100-220 \%$, and $83,982.8$ in the strain range of $220-300 \%$; these metrics satisfied the demands for monitoring various fine and large deformations. By combining the cracking mechanism and tunneling effect, Chen et al. [37] designed a sensor with an ultra-low detection limit of 0.01 and an ultra-wide detection range $(>100 \%)$ - it also had an excellent/fast response and good repeatability. With these properties, sensors can be used to detect subtle physiological activities (pulses, coughing, and swallowing) as well as walking, leg lifting, and squatting. However, these reported methods require time-consuming procedures and wastage of materials, limiting the commercialization of flexible strain sensors.

$3 \mathrm{D}$ printing is an advanced manufacturing strategy widely used in electronics, biomedicine, and aerospace. If offers low processing costs, high manufacturing accuracy, and excellent productivity. Research has been devoted to the application of 3D printing technology to flexible strain sensor manufacturing [38]. 3D printing technology offers a promising approach to fabricate flexible strain sensors compared with conventional manufacturing approaches. Currently, processing methods based on fused deposition modeling (FDM) and direct ink writing (DIW) are widely used to build strain sensors. Christ et al. [39] fabricated bidirectional strain sensors using a nozzle to print TPU as a substrate and another nozzle to print a mixture of TPU and MWCNT as a conductive filler. Wajahat et al. [40] prepared flexible strain sensors via a CNT ink formulated from multiwall nanotubes (MWNTs) and polyvinylpyrrolidone (PVP). The sensors exhibited high stability with a gauge factor of 13.07. These could be applied to human motion monitoring and remote monitoring of robotic devices. However, the raw material used in FDM technologies must be pre-processed under high temperature before printing, and the surface of the printed component is rough. The DIW 3D printing approach can easily clog the nozzle, and the component requires post-processing.

In this study, an efficient, low-cost, and mass-produced EFD micro-scale 3D printing [41-46] and molding hybrid method is proposed to fabricate PDMS channel films. MWCNTs were filled into PDMS films with channel networks to prepare flexible strain sensors with high sensitivity, transparency, and stretchability. Three different ratios of PDMS prepolymer and cross-linker (10:1, 15:1, and 20:1) were chosen to test the sensitivity and stretchability of the strain sensor, and the optimal ratio was chosen to investigate the detection range, sensitivity, stability, transparency, and responsiveness of the sensor. The final fabricated flexible strain sensors with up to $84 \%$ transparency exhibited ultra-high sensitivity (gauge factor up to 1500 at 20\% tensile strain) and over 8000 stretch-release cycles. The feasibility and effectiveness of the sensor were demonstrated by mounting the sensor on various parts of the human body for testing. The proposed EFD micro-scale 3D printing method opens up new prospects for the fabrication of flexible transparent strain sensors for practical applications in various fields. 


\section{Materials and Methods}

\subsection{Materials}

The MWCNT dispersion (XFWPM-H-M31) was purchased from XFNANO Materials Tech Co., Ltd., Nanjing, China, and dispersed in water. The concentration of the MWCNT was $14 \mathrm{wt} . \%$. The length of the CNT was approximately $10 \mu \mathrm{m}$, and the outside diameter was greater than $50 \mathrm{~nm}$. The PDMS prepolymer and cross-linker (Sylgard 184) were purchased from Dow Corning, Midland, MI, USA. Conductive silver ink (CON-EHD50) purchased from BroadTeko, Beijing, China. CON-EHD50 is a nano-silver conductive ink custom developed for electrohydraulic inkjet printing (EHD jetting, also known as near-field electrospinning).

\subsection{Fabrication of the Flexible PDMS Film with Channel Structures}

A 20- $\mu$ m-thick layer of liquid PDMS (prepolymer to cross-linker ratio of 10:1; uniformly mixed and vacuumed) was spin-coated on the glass surface and cured for 30 min to form a hydrophobic surface. Polymer printing generally adopts FDM technology, which requires pre-treatment at high temperature. The intersection points cannot be lapped when printing the grid structure, which is unfavorable for PDMS films with manufactured channel structures [41]. Silver grid printing has good consistency and neat intersections and is a simple process, which is suitable for master molds of PDMS films with channel structure manufacturing [42]. The silver grid structure (width of $20 \mu \mathrm{m}$; five layers) was deposited on the hydrophobic glass surface using EFD microscale 3D printing technology and cured at $100{ }^{\circ} \mathrm{C}$ for $30 \mathrm{~min}$ to enhance the hardness of the silver paste structure (Figure 1a). The liquid PDMS prepolymer and cross-linker were uniformly mixed at a ratio of 15:1 (10:1 or 20:1) and degassed for 30 min under vacuum. Afterward, the mixture was drop cast onto PDMS films with a metallic silver grid and then cured on a hot plate at $100{ }^{\circ} \mathrm{C}$ for $30 \mathrm{~min}$ (Figure 1b). The PDMS film with channel structures was peeled off from the PDMS substrates after curing to yield a PDMS channel structure film with flexibility and transparency (Figure 1c).

(a)

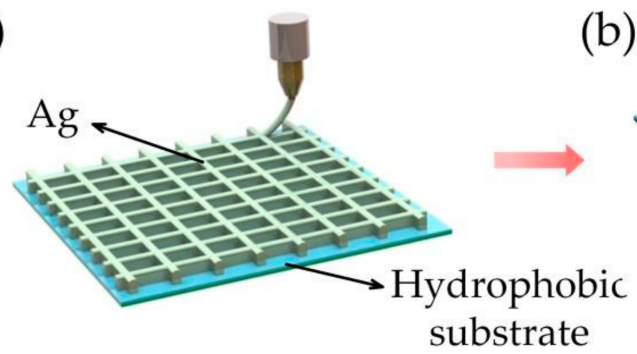

(b)
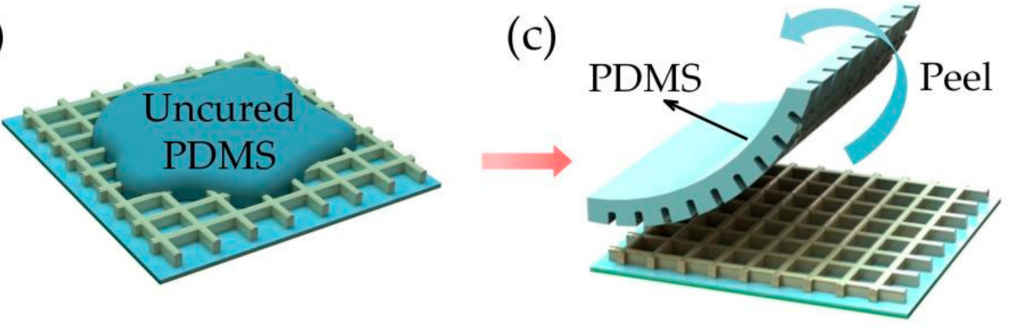

$\checkmark$

(f)

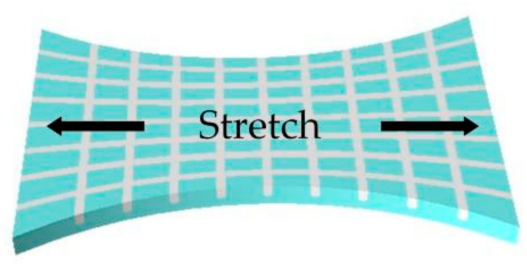

(e)

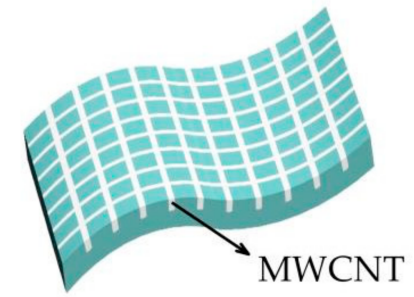

(d)

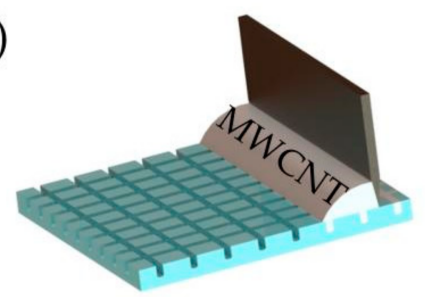

Figure 1. Fabrication process of the flexible strain sensor with the embedded MWCNT network.

\subsection{Fabrication of the Transparent Strain Sensor with an Embedded MWCNT Network}

The MWCNT was chosen as the sensing material due to good electrical properties and chemical stability $[47,48]$. Figure S1 shows the TGDSC curves of MWCNTs. There is a strong heat absorption peak on the DSC curve at around $100^{\circ} \mathrm{C}$, which is the evaporation of heavy 
adsorbed water of the sample. There is a more obvious weight loss on the corresponding TG curve, indicating the complete evaporation of water from MWCNTs after $7 \mathrm{~min}$ of drying at $100{ }^{\circ} \mathrm{C}$. In the range of $100-360^{\circ} \mathrm{C}$, the TG curve tends to be stable, and after exceeding $360^{\circ} \mathrm{C}$, there is a weight loss on the TG curve, indicating that there is no significant change in the sample weight of MWCNT in the range of $100-360^{\circ} \mathrm{C}$, and the conductivity of MWCNT is not affected in this temperature range. Due to the chosen MWCNT solution is aqueous, and PDMS is hydrophobic; thus, the surface of the PDMS films was hydrophilically treated with vinyltriethoxysilane solution ( $1 \%$ vinyltriethoxysilane dispersed in water and soaked for $2 \mathrm{~h}$ ). The MWCNT solution was then poured on the side surface of the PDMS and dragged at a constant speed with a blade. During dragging, the MWCNT solution was filled into the PDMS channel due to the capillary forces (Figure 1d). The MWCNT in the channel was dried on a hot plate at $70^{\circ} \mathrm{C}$ for $3 \mathrm{~min}$, and the excess MWCNT was removed from the PDMS membrane surface by ethanol-assisted scraping. The embedded MWCNT-conducting network was fabricated inside the PDMS channels (Figure 1e). To obtain a reliable electrical connection, a thin layer of silver ink was first spread on the two ends of the conductive route, followed by curing at $70{ }^{\circ} \mathrm{C}$ for $1 \mathrm{~h}$ to decrease the contact resistance. Later, a thin and flexible conductive tape was pasted on the silver ink, and there was a thin layer of liquid metal between the conductive tape and the silver ink, resulting in a reliable electrical connection. Finally, the contact points were encapsulated by the PDMS, thereby providing protection for reliable operation.

\subsection{Measurement and Characterizations}

To test the strain sensor performance of the embedded MWCNT network, the sensor film was stretched to different strains using a tensile tester, and the resistance changes were measured using a resistance tester (Applent, Edison, NJ, USA, AT516). A UV-Vis spectrophotometer (METASH, Shanghai, China, UV-6100) was used to measure the optical transmittance of the sensor. The structural morphology of the channel crack structure was characterized using a field emission scanning electron microscope (SEM) (SU8010, HITACHI, Tokyo, Japan).

\section{Results and Discussion}

\subsection{Performance Characterizations of the Transparent Strain Sensor with the Embedded MWCNT Network}

The width of the non-transparent channel was set at $20 \mu \mathrm{m}$ to obtain relatively good conductivity and proper transparency of the strain sensor. For printing metallic silver meshes, a width of $20 \mu \mathrm{m}$ is more suitable and easier to print. Meanwhile, the size of the channel spacing was kept to $1000 \mu \mathrm{m}$. When the channel width was constant, a smaller channel spacing led to poor transparency. Therefore, a spacing of $1000 \mu \mathrm{m}$ was selected to fabricate the sensor to ensure good transparency. Meanwhile, the PDMS prepolymer and cross-linker ratios directly affect the tensile performance and sensitivity of the strain sensors and also impact the conductivity of the sensor. Therefore, experiments to test the different ratios of PDMS prepolymer and cross-linker were performed (Figure 2). The ratio of PDMS prepolymer and cross-linking agent commonly used in general experiments is 10:1. Figure 2a(i-iii) shows the filling effect of MWCNT with PDMS prepolymer and crosslinker ratios of 10:1, 15:1, and 20:1, respectively. The results show that MWCNT is well-filled at the 10:1 ratio with no obvious cracks in the channel; the MWCNT exhibits few cracks at the 15:1 ratio, and the MWCNT is less filled at the 20:1 ratio with more obvious cracks in the channel. The ratio of PDMS has a strong influence on the interfacial stability of the conductive structures; the stability of the channel network improves as the PDMS ratio increases, thus effectively filling the MWCNT into the channel as the blade cuts across the channel surface. The PDMS becomes soft when the PDMS ratio is too large, causing the channel to close when the blade cuts across the channel. The MWCNT crack expansion in the channel under the same stretching condition for different PDMS ratios is shown in Figure 2b. It is obvious that the MWCNT crack expansion is smaller 
at the 10:1 ratio (Figure 2b(i)), and the MWCNT crack expansion is the largest at the 20:1 ratio(Figure $2 \mathrm{~b}(\mathrm{iii})$ ). On the one hand, the filling amount of MWCNT has an effect on the crack extension during stretching. Figure S2 shows the dispersion of MWCNT in the matrix and the microscopic morphology of MWCNT filling in the PDMS channel. It can be seen that MWCNT is well dispersed in the matrix, MWCNT has a high aspect ratio, and the fibrous MWCNT can effectively form a bridge structure to support the connection between cracks when stretched. The more MWCNT filling, the slower the crack formation when stretched, which enables the sensor to withstand greater tensile strain. On the other hand, the PDMS channels have a protective effect on the MWCNT structure, and PDMS with increasing ratio of prepolymer and cross-linker becomes softer, leading to a decrease in the protective effect of the channels on the MWCNT structure.

(a)
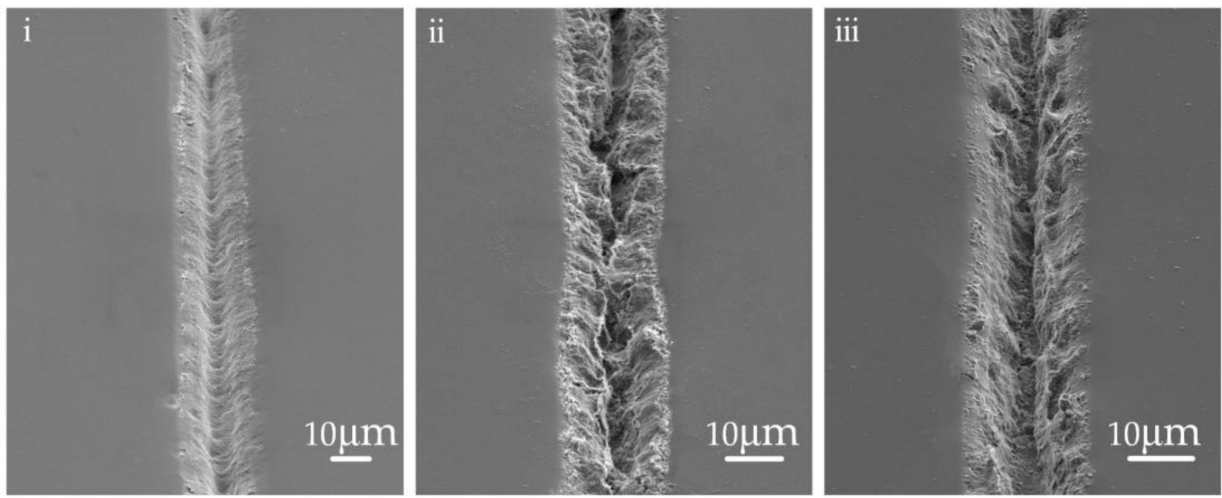

(b)

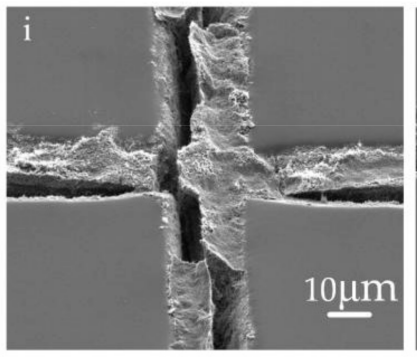

(c)

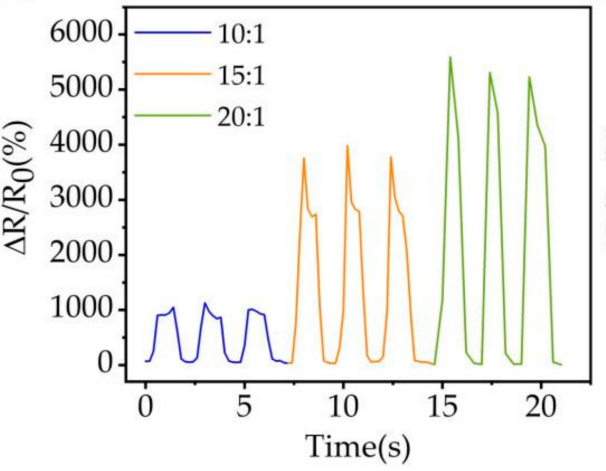

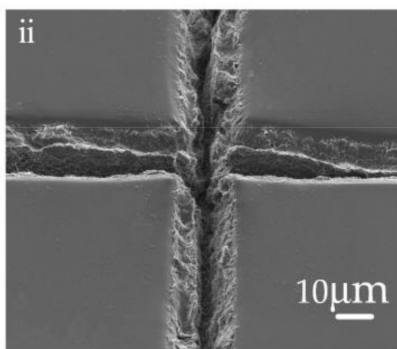

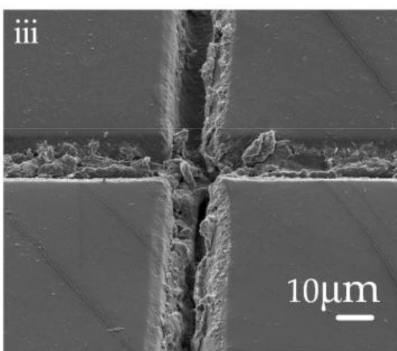

(d)

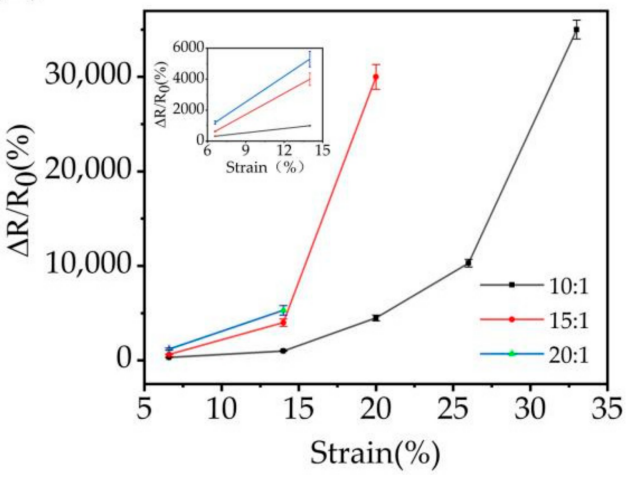

Figure 2. Performance of sensors prepared with different ratios of PDMS prepolymer and crosslinker. (a) MWCNT filling effect of PDMS films prepared with different ratios: 10:1, 15:1, and 20:1. (b) Stretching effect after filling MWCNT with PDMS films of different proportions. (c) Variation of $\Delta R / R_{0}$ at $14 \%$ tensile strain for sensors prepared with different PDMS ratios. (d) Maximum tensile strain of the sensors prepared with different PDMS ratios.

The mechanism of flexible strain sensor with embedded MWCNT network is mainly derived from crack propagation. Tensile strain changes the crack structure in the chan- 
nel. The reconnection or disconnection of the crack structure fundamentally changes the conductivity of the strain sensor. Thus, the sensitivity of the sensor becomes larger with increasing ratio of PDMS prepolymer to cross-linker. The relative resistance changes of sensors prepared with different proportions of PDMS prepolymer and cross-linking agent under a tensile strain of $14 \%$ are shown in Figure 2c. The normalized resistance $\left(\Delta R / R_{0}\right)$ is defined as the ratio between the resistance change $(\Delta R)$ to the initial resistance $\left(R_{0}\right)$, where $\Delta \mathrm{R}=\mathrm{R}-\mathrm{R}_{0}$.

It can be seen that the change in normalized resistance gradually becomes larger as the PDMS prepolymer to cross-linker ratio increases. The normalized resistance value is 1000 , below a ratio of 10:1. The $\Delta R / R_{0}$ increases to 4000 when the ratio increased to $15: 1$, and the maximum value is 5300 when the ratio is 20:1. The high sensitivity of the sensor is achieved by the disconnection and reconnection of the MWCNT structure. At the same tensile strain, the filling amount of MWCNT with a 10:1 ratio of PDMS prepolymer to cross-linker is significantly better than the filling amount of MWCNT with a 15:1 and 20:1 ratio of PDMS prepolymer to cross-linker. Therefore, the fibrous MWCNT can achieve bridge lap during the tensile process, resulting in insignificant changes in the sensor resistance value, but the stretching range of the sensor gets improved. Figure $2 \mathrm{~d}$ shows the maximum tensile strain that the PDMS can withstand at different ratios. The results show that the sensor prepared with a PDMS ratio of 20:1 can only withstand $14 \%$ of the tensile strain, while the sensor prepared with a PDMS ratio of 10:1 can withstand a maximum strain of $33 \%$ (20\% at 15:1). The PDMS ratio impacts both the resistance change and the tensile performance of the sensor. Although the sensor prepared with a PDMS ratio of 20:1 has high sensitivity, it can only withstand a maximum of $14 \%$ tensile strain. Due to the protective effect of PDMS channels on MWCNT, when the ratio of PDMS prepolymer and cross-linker is 10:1, the MWCNT cracks in PDMS channels are more stable, the fiber structure of MWCNT can form a bridge structure within $30 \%$ of the tensile strain. When the ratio of PDMS prepolymer and cross-linker was 20:1, the PDMS channel network became soft, and the protective effect of PDMS channel network on MWCNT was reduced during stretching, resulting in the fiber structure of MWCNT being destroyed when the tensile strain exceeded $14 \%$. Therefore, the dynamic stability is poor at $14 \%$ strain, and the sensor can only be applied to the motion monitoring of the human body with a strain below $14 \%$. The sensitivity of the sensor prepared with a PDMS ratio of 10:1 is relatively low-it has a tensile strain of 30\% and is used for the human motion monitoring of a strain below $30 \%$. The sensor prepared with a PDMS ratio of 15:1 has good sensitivity and can monitor human motion with a strain below $20 \%$. The ratio of PDMS prepolymer and cross-linker has a dual influence on the sensitivity and detection range; thus, a comprehensive consideration should be made for practical applications.

Considering the tensile strain and sensitivity performance of the sensor, a 15:1 ratio of PDMS mixture was used to prepare the flexible strain sensor with the embedded MWCNT network. Figure 3a,b shows the macroscopic images of the fabricated sensor in different states, respectively, illustrating its good flexibility and transparency.

The PDMS films were subjected a hydrophilic treatment to obtain a better filling effect of MWCNT. After the hydrophilic treatment, a thin layer of milky substance appeared on the surface of the PDMS film, which impacted the optical transmittance of the sensor. Therefore, we tested the optical transmittance of hydrophilic-treated and non-hydrophilictreated sensors separately (Figure 3c). The transmittance of the sensor without hydrophilic treatment was $87 \%$, which was slightly reduced to $84 \%$ after hydrophilic treatment. The MWCNT filling effect of the PDMS channel film without hydrophilic treatment is poor and requires multiple scrapings (Figure 3d(ii)). Whereas the filling rate of MWCNT of the hydrophilic-treated PDMS film was significantly increased (Figure 3d(i)), which improves the conductivity of the sensor. Therefore, the preparation of PDMS films subjected to hydrophilic treatment with flexible strain sensor with an embedded MWCNT network can guarantee good conductivity and appropriate light transmittance. 
(a)

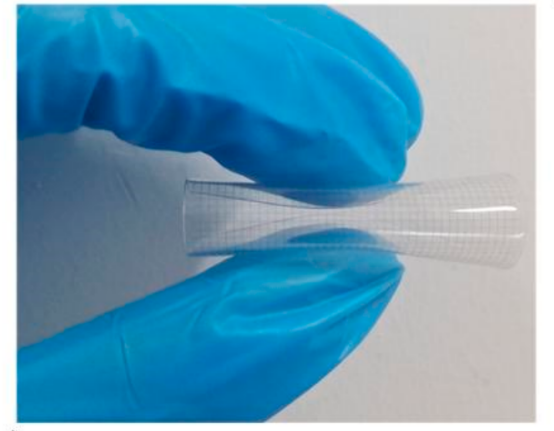

(c)

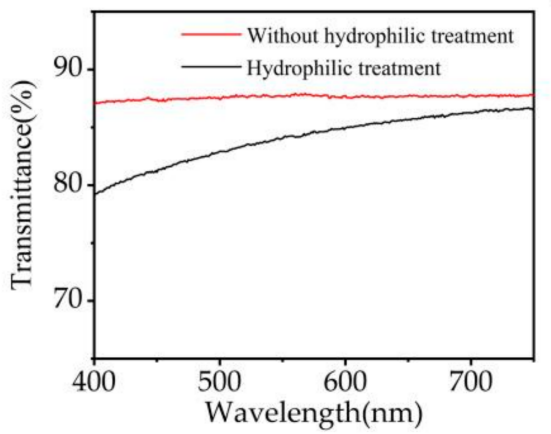

(b)

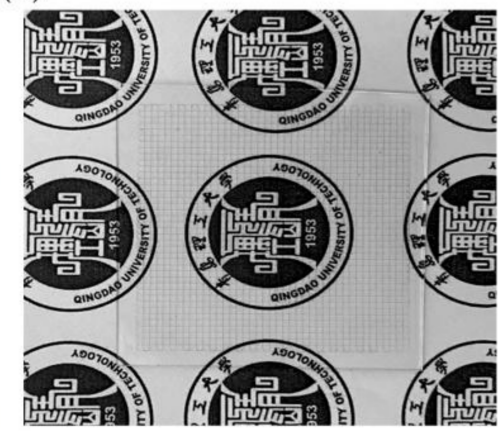

(d)

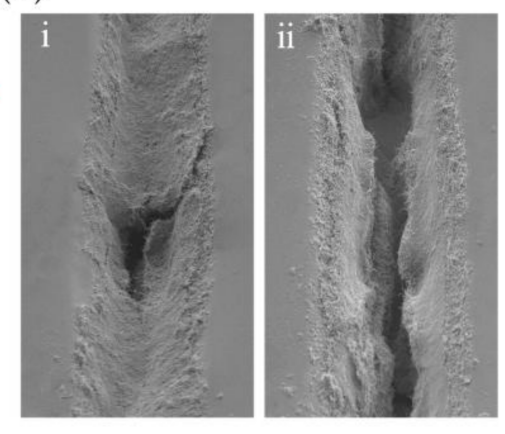

Figure 3. (a) Snapshot of the strain sensor after bending. (b) Snapshot of the strain sensor located on the logo of Qingdao University of Technology. (c) Light transmission rate of strain sensors without hydrophilic treatment and with hydrophilic treatment. (d) MWCNT filling effect of hydrophilictreated PDMS films and non-hydrophilic-treated PDMS films.

To obtain better detection sensitivity, and be able to apply the sensor to multiple parts of the human body for monitoring. A PDMS prepolymer and cross-linker ratio of 15:1 was selected to prepare the flexible strain sensor with embedded MWCNT networks; the channel width was $20 \mu \mathrm{m}$, depth was $30 \mu \mathrm{m}$, and channel spacing was $1000 \mu \mathrm{m}$. The performance of the strain sensor is shown in Figure 4. The gauge factor (GF) is an important parameter to evaluate the performance of the sensor and defines the sensitivity of the sensor. Figure 4a illustrates the typical response of the strain sensor to different strains. The GF values of the strain sensors ranges from 10 to 1500 when the strain increased from $6.6 \%$ to $20 \%$; the GF of the strain sensor reaches 285 at $14 \%$ strain, which are comparable to those of the MWCNT based strain sensors in most reported work [2,9,10,12,28,48] (Table 1), and the sensors manufactured in this work have better mechanical stability than other sensors with transparency. The cracking of the strain sensor is maximized when the strain exceeds $20 \%$ resulting in fracture of the MWCNT structure, and the resistance reaches zero. Meanwhile, the normalized resistance variation is unstable in the $20 \%$ strain range. Then, to conduct a more in-depth study on the performance of the sensor, the response time of the sensor was tested. Figure $4 \mathrm{~b}$ shows that the sensor was subjected to a stretch-release cycle at $14 \%$ strain, and the strain was applied at $4 \mathrm{~mm} \mathrm{~s}^{-1}$ and held for $8 \mathrm{~s}$ before being released. The experimental results show that the manufactured strain sensors have a response time and recovery time of $600 \mathrm{~ms}$, indicating a good response and recovery time, which has good potential for applications in human motion detection. 
(a)

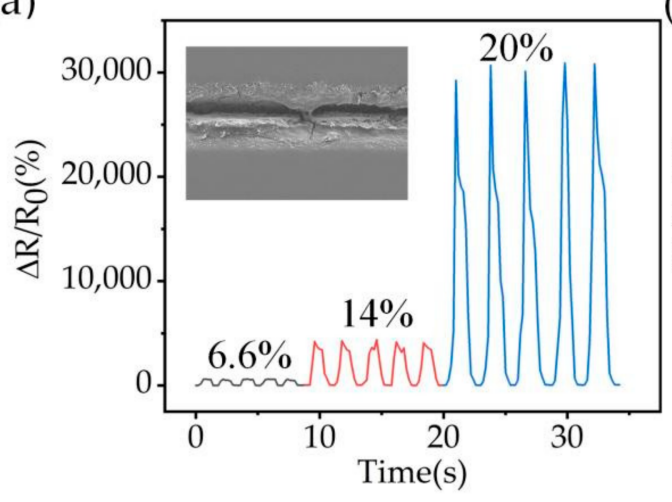

(b)

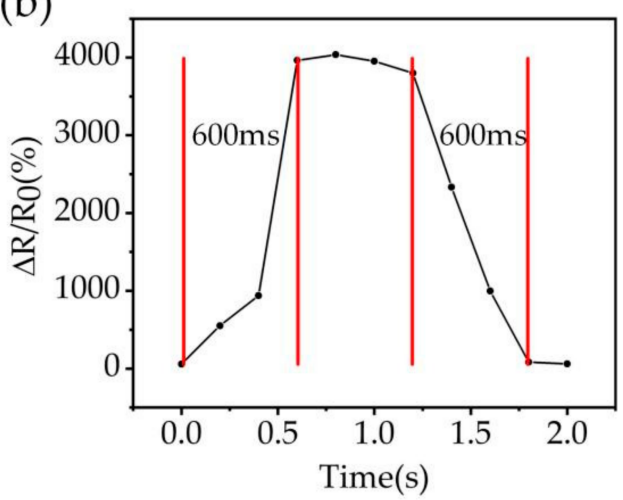

Figure 4. Sensor performance at PDMS ratio of 15:1. (a) Normalized resistance of the strain sensor at $6.6 \%, 14 \%$, and $20 \%$ strain. The inset shows the SEM of crack extension after stretching MWCNT

(b) Response/recovery time of the sensor at a strain of $14 \%$.

Table 1. Summary of performance results of carbon-based strain sensors recently reported.

\begin{tabular}{|c|c|c|c|c|c|}
\hline References & Materials & Strain Range & GF & Mechanical Stability & Transmittance \\
\hline This work & MWCNTs/PDMS & $20 \%$ & $\begin{array}{c}90(6.6 \%) \\
285(14 \%) \\
1500(20 \%)\end{array}$ & 8000 cycles at $14 \%$ strain & $84 \%$ \\
\hline [2] & SWCNTs/CB/PDMS & $300 \%$ & $\begin{array}{c}0.91(0-100 \%) \\
3.25(100-255 \%) \\
13.1(255-300 \%)\end{array}$ & 2500 cycles at $200 \%$ strain & $\mathrm{N} / \mathrm{A}$ \\
\hline [9] & CNTs/Ecoflex & $20 \%$ & $14.5(0-20 \%)$ & 50 cycles at $20 \%$ strain & $\mathrm{N} / \mathrm{A}$ \\
\hline [10] & SWCNTs/PDMS & $280 \%$ & $\begin{array}{c}0.82(0-40 \%) \\
0.06(60-200 \%)\end{array}$ & 10,000 cycles at $200 \%$ strain & $\mathrm{N} / \mathrm{A}$ \\
\hline [12] & CNTs/Graphene/PDMS & $120 \%$ & $\begin{array}{c}182.5(0-3 \%) \\
45.6(3-57 \%) \\
70.2(57-90 \%) \\
186.5(90-120 \%)\end{array}$ & 10,000 cycles at $40 \%$ strain & $\mathrm{N} / \mathrm{A}$ \\
\hline [28] & CNTs/PDMS & $30 \%$ & $\begin{array}{c}3(0-10 \%) \\
4(10-20 \%) \\
6.6(20-30 \%)\end{array}$ & 1000 cycles at $40 \%$ Strain & $92 \%$ \\
\hline [48] & MWCNTs/PDMS & $10 \%$ & $\begin{array}{c}17.6(0-5 \%) \\
134.1(5-8.75 \%) \\
330(10 \%)\end{array}$ & 2000 cycles at $7 \%$ strain & $87 \%$ \\
\hline
\end{tabular}

The mechanical stability of strain sensors is another important parameter for evaluating the performance of strain sensors. In practical applications, the sensor needs to undergo thousands of stretch-release processes to maintain almost constant resistance. The mechanical stability of the flexible strain sensor with the embedded MWCNT network was investigated using a homemade tensile test platform. The stretch-release process of the sensor is shown in the Figure 5a. The sensor is fixed on a tensile tester, and the resistance change of the sensor in the range of $0-14 \%$ of tensile strain is measured using a resistance tester, and the measured data is transmitted to the PC for processing. Figure 5a(i,ii) shows the resistance values of the sensor measured at $0 \%$ and $14 \%$ of the tensile strain, respectively. Figure $5 b$ shows the relative resistance changes of the sensor under stretch-release cycles. The sensing stability was tested continuously for 8000 stretch-release cycles at $14 \%$ strain. The $\Delta R / R_{0}$ always varies from $0 \%$ to $4200 \%$, while the resistance always returns to the initial state after release. Figure $5 c$,d shows the $\Delta R / R_{0}$ changes during $500-520$ and $7500-7520$ stretch-release cycles. After 8000 cycles, the $\Delta R / R_{0}$ only has a small increase, 
and the sensor remains stable. The channel structure of the sensor provides good interfacial stability for MWCNT crack stretching. Since the MWCNT is deposited inside the PDMS channel and forms a three-sided contact with PDMS, compared with other 2D MWCNT deposited on the substrate surface, the MWCNT in this paper forms a 3D structure, which ensures good interfacial contact. Meanwhile, the crack is fully extended and no generated new cracks after a certain tensile strain allowing the sensor resistance change to be stable in the $0-4200 \%$ interval.

(a)

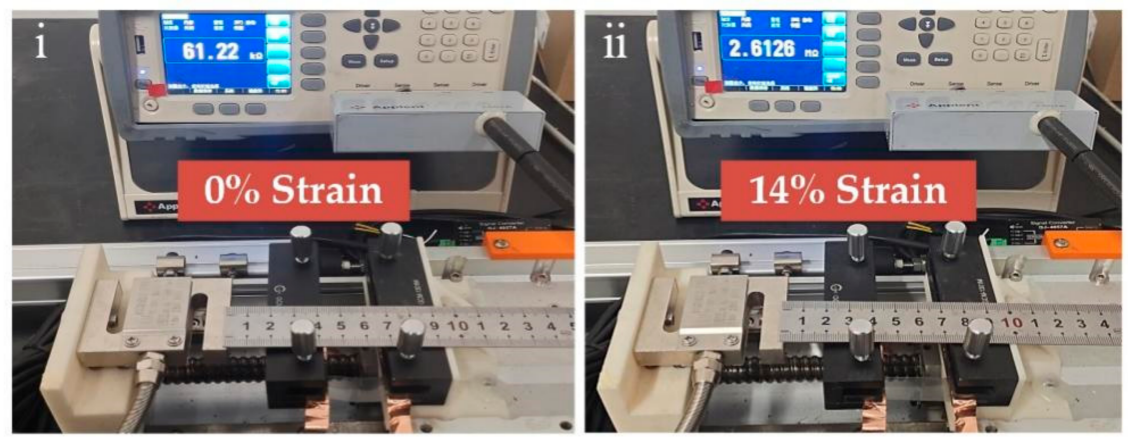

(b)

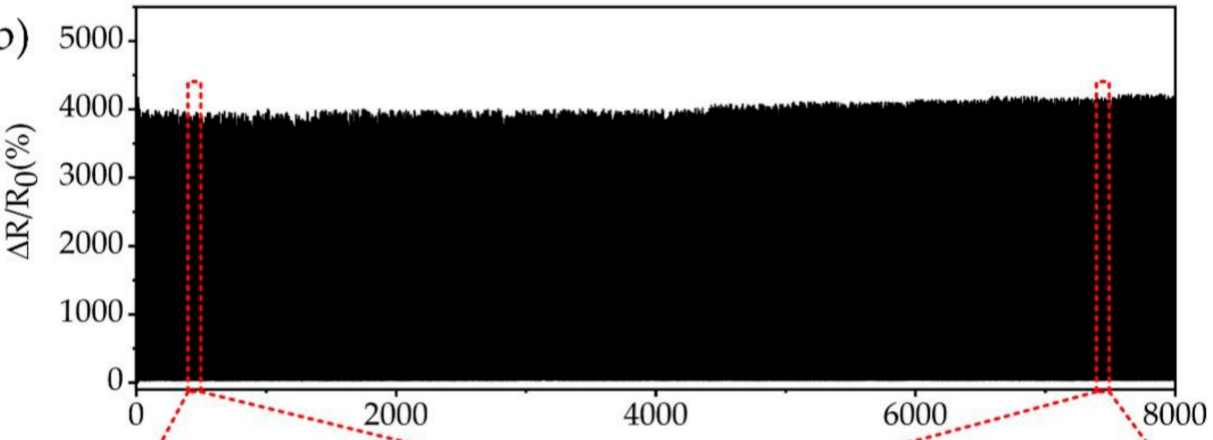

(c)

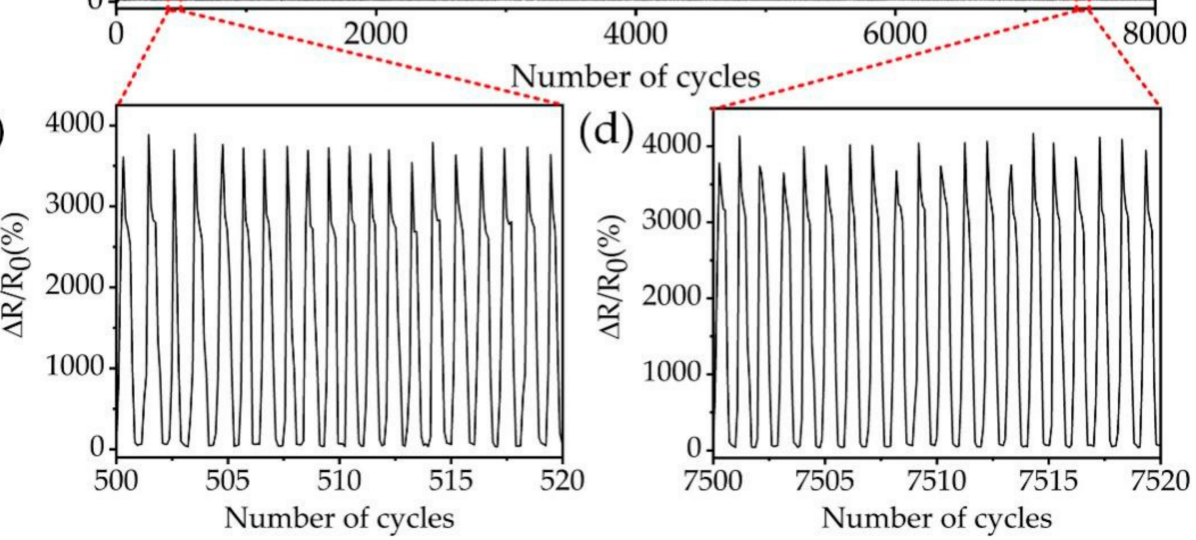

Figure 5. Stability performance of the strain sensor with the embedded MWCNT network. (a) Resistance changes of the sensor during 10,000 stretch-release cycles. (b) Resistance changes between 500 and 520 stretch-release cycles. (c) Resistance changes between 7500 and 7520 stretch-release cycles. (d) Resistance changes at $0 \%$ and $14 \%$ strain.

\subsection{Applications of the Transparent Strain Sensor with the Embedded MWCNT Network}

The flexible strain sensors based on PDMS prepolymer and cross-linker ratio of 15:1 show high sensitivity and transparency. The optical transparency of the sensor reduces any inconvenience to the user while wearing it during normal activity. This increases the acceptance of wearable devices, allowing the sensor to be more useful in terms of applications. The strain sensor with the embedded MWCNT network could be potentially useful in wearable devices, biomedicine, and health monitoring.

To evaluate the possibility of sensor applications in wearable devices, tests were conducted on finger or wrist flexion, swallowing movements, changes in eye movements and facial expressions, and changes in neck flexion (Figure 6). To isolate the human body 
resistance from the measurement results, medical tape or insulating tape was used to adhere the strain transducer to the human skin surface. When the sensor is attached to the wrist and finger (Figure $6 a, b$ ), it can clearly detect the changes in resistance when the wrist and finger are bent. The sensor identifies different resistance changes; the normalized resistance value of the wrist reaches $8000 \%$ and that of the finger is $4000 \%$. Meanwhile, subtle human motion such as swallowing and eye movements can also be promptly and accurately monitored by the strain sensor (Figure $6 c, d$ ). The $\Delta R / R_{0}$ responses reached $70 \%$ or more with human swallowing.

(a)

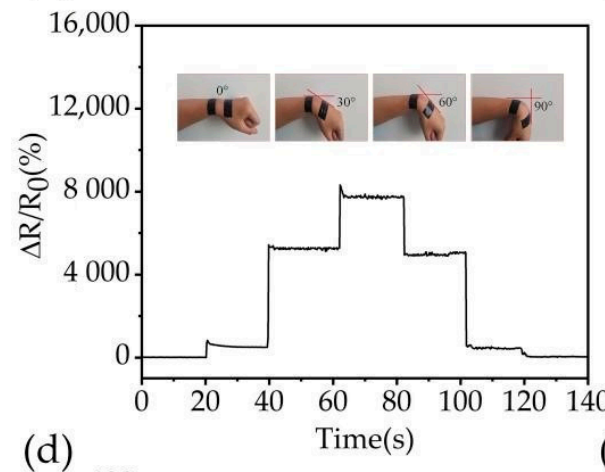

(d)

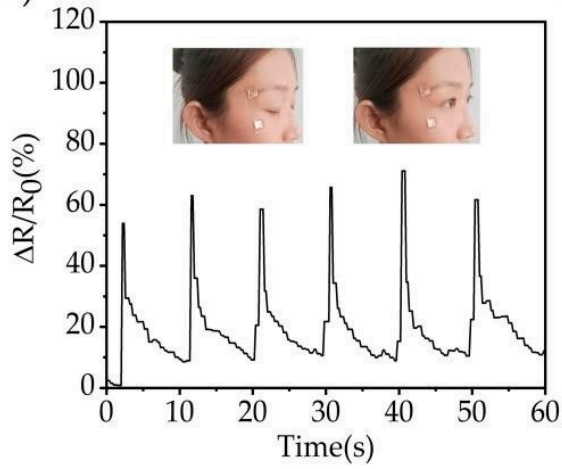

(b)

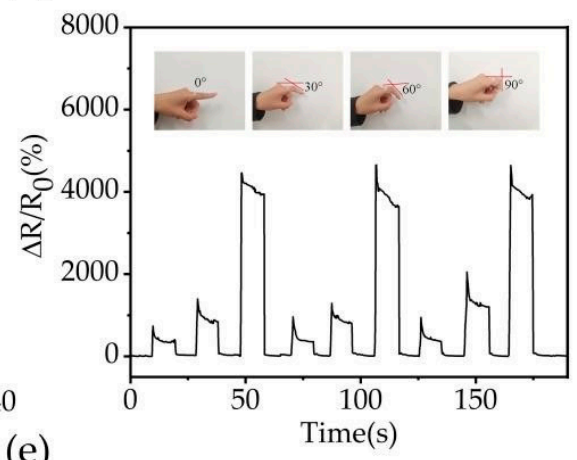

(e)

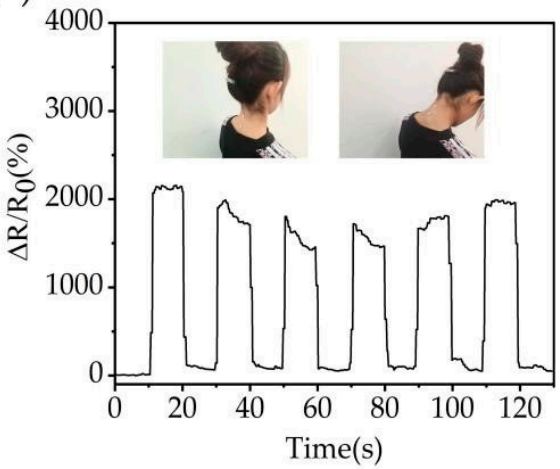

(c)

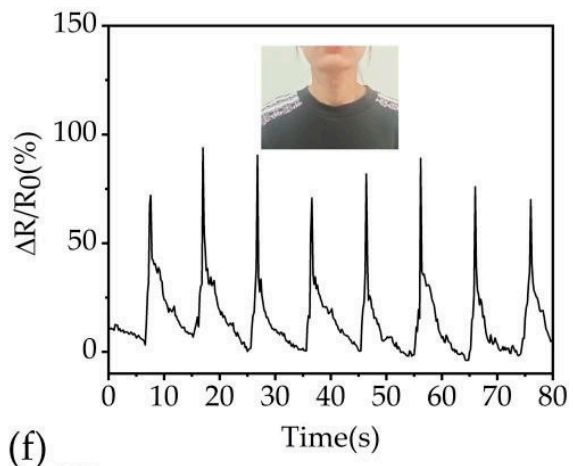

(f)

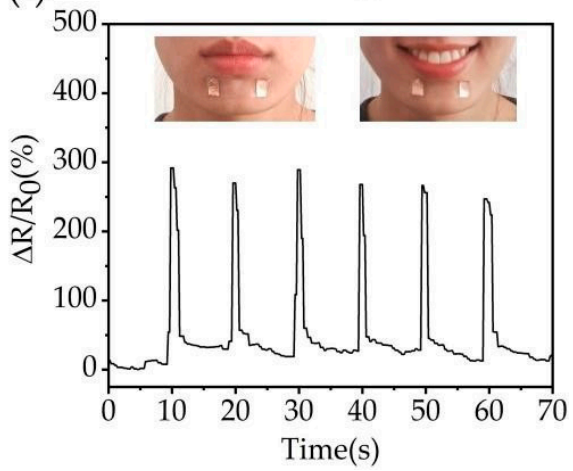

Figure 6. Applications of the strain sensor with the embedded MWCNT network in monitoring various human motions. Change of $\Delta \mathrm{R} / \mathrm{R}_{0}$ for: (a) bending of wrist, (b) bending of finger, (c) swallowing of food, (d) blinking, (e) bending of neck, and (f) smiling.

Due to the development of technology, daily life is inseparable from high-tech products such as mobile phones and computers. "Phubbing" has become synonymous with people, and prolonged bending of the neck is dangerous to human health. The sensor can monitor the neck posture: the resistance tends to be stable when the neck is upright, but $\Delta R / R_{0}$ increases to $2000 \%$ when the neck is bent (Figure 6e). Moreover, the sensor can quickly respond to signal changes. Due to their high sensitivity and fast response times, the proposed flexible strain sensor with an embedded MWCNT network can improve "Phubbing". Furthermore, the sensor can also be used to monitor changes in human facial expressions by mounting the sensor on the chin (Figure 6f). When smiling, the muscles in the chin stretch to generate strain in the sensor, thus resulting in a change in the normalized resistance values. Human facial activity can be determined by analyzing the change in resistance values. These results obviously indicate that the strain sensor with the embedded MWCNT network has good responsiveness, high sensitivity, and high transparency. In addition, the sensor can also be applied to small and medium deformation tests and has the potential for use in real-time medical treatments. 


\section{Conclusions}

In summary, we have successfully fabricated a transparent strain sensor with an embedded MWCNT network to monitor human movement. The embedded PDMS channel film was fabricated via an EFD microscale 3D printing and molding hybrid process free of time-consuming procedures and material wastage. Meanwhile, the PDMS channel film has the advantage of high degree of freedom, and is suitable for filling other types of conductive materials, and the performance of the sensor can also be improved by designing different structures. The sensor was based on the cracking mechanism and exhibited excellent characteristics in terms of sensitivity, transparency, stability, and response time. The sensing performance of the flexible strain sensor was systematically characterized, and the sensitivity of the sensor could be improved by changing the ratio of PDMS prepolymer and cross-linker (10:1, 15:1, and 20:1). The detection range could thus be reduced. Meanwhile, the PDMS channel network provides good protection for MWCNTs. The resulting sensor shows high sensitivity (the GF of 90 at a strain of $6.6 \%$, the GF of 285 at a strain of $14 \%$, the GF of 1500 at a strain of 20\%), stability (8000 cycles), responsiveness, and optical transparency $(>84 \%)$. These features make the sensor promising for various human motiondetection applications. Furthermore, the manufacturing of sensors using EFD 3D printing and molding hybrid process offers high efficiency and low cost, which are suitable for mass production. The proposed sensor manufacturing method offers a new technological route for the application of 3D printing technologies in wearable electronic devices.

Supplementary Materials: The following are available online at https:/ / www.mdpi.com/article/ 10.3390/nano12010120/s1, Figure S1: TGDSC image of MWCNTs, Figure S2: SEM of the dispersion of MWCNTs unfilled in PDMS channel and the dispersion of MWCNTs filled PDMS channel.

Author Contributions: Conceptualization, H.L. (Hongke Li); methodology, R.W., L.S. and S.S.; software, W.G.; validation, H.L. (Hongbo Lan); formal analysis, H.L. (Hongbo Lan); investigation, L.S. and S.S.; resources, L.S.; data curation, R.W.; writing-original draft preparation, R.W.; writing-review and editing, X.Z.; visualization, W.G.; supervision, H.L. (Hongke Li); project administration, X.Z. and H.L. (Hongbo Lan); funding acquisition, X.Z. and H.L. (Hongbo Lan). All authors have read and agreed to the published version of the manuscript.

Funding: This study was supported by the National Natural Science Foundation of China (Grant No. 52175331), the Support plan for Outstanding Youth Innovation Team in Universities of Shandong Province, China (Grant No. 2020KJB003), the Major Basic Research plan of the National Natural Science Foundation of Shandong Province (Grant No. ZR2020ZD04), and the Shandong Provincial Natural Science Foundation (Grant No. ZR2021ME139). The authors thank LetPub (www.letpub.com, accessed on 29 November 2021) for its linguistic assistance during the preparation of this manuscript.

Institutional Review Board Statement: The study was conducted in accordance with the Declaration of Helsinki, and approved by the Institutional Review Board (or Ethics Committee) of Health Commission of Shandong Province, China (protocol code: 12370000495540761M, date of approval: 20 December 2021). for studies involving humans.

Informed Consent Statement: Informed consent was obtained from all subjects involved in the study.

Data Availability Statement: Data sharing is not applicable to this article.

Conflicts of Interest: The authors declare no conflict of interest.

\section{References}

1. Sun, Z.; Yang, S.; Zhao, P.; Zhang, J.; Yang, Y.; Ye, X.; Zhao, X.; Cui, N.; Tong, Y.; Liu, Y.; et al. Skin-like Ultrasensitive Strain Sensor for Full-Range Detection of Human Health Monitoring. ACS Appl. Mater. Interfaces 2020, 12, 13287-13295. [CrossRef]

2. Zheng, Y.; Li, Y.; Dai, K.; Wang, Y.; Zheng, G.; Liu, C.; Shen, C. A highly stretchable and stable strain sensor based on hybrid carbon nanofillers/polydimethylsiloxane conductive composites for large human motions monitoring. Compos. Sci. Technol. 2018, 156, 276-286. [CrossRef]

3. Yang, Z.; Wang, D.Y.; Pang, Y.; Li, Y.X.; Wang, Q.; Zhang, T.Y.; Wang, J.B.; Liu, X.; Yang, Y.Y.; Jian, J.M.; et al. Simultaneously Detecting Subtle and Intensive Human Motions Based on a Silver Nanoparticles Bridged Graphene Strain Sensor. ACS Appl. Mater. Interfaces 2018, 10, 3948-3954. [CrossRef] [PubMed] 
4. $\quad$ Lee, S.W.; Park, J.J.; Park, B.H.; Mun, S.C.; Park, Y.T.; Liao, K.; Seo, T.S.; Hyun, W.J.; Park, O.O. Enhanced Sensitivity of Patterned Graphene Strain Sensors Used for Monitoring Subtle Human Body Motions. ACS Appl. Mat. Interfaces 2017, 9, 11176-11183. [CrossRef]

5. $\quad$ Eom, J.; Jaisutti, R.; Lee, H.; Lee, W.; Heo, J.S.; Lee, J.Y.; Park, S.K.; Kim, Y.H. Highly Sensitive Textile Strain Sensors and Wireless User-Interface Devices Using All-Polymeric Conducting Fibers. ACS Appl. Mater. Interfaces 2017, 9, 10190-10197. [CrossRef]

6. Yang, D.; Wang, H.; Luo, S.; Wang, C.; Zhang, S.; Guo, S. Paper-Cut Flexible Multifunctional Electronics Using MoS2 Nanosheet. Nanomaterials 2019, 9, 922. [CrossRef]

7. Wang, S.; Fang, Y.; He, H.; Zhang, L.; Li, C.A.; Ouyang, J. Wearable Stretchable Dry and Self-Adhesive Strain Sensors with Conformal Contact to Skin for High-Quality Motion Monitoring. Adv. Funct. Mater. 2020, 31, 2007495. [CrossRef]

8. Yoon, S.; Kim, H.K. Cost-effective stretchable Ag nanoparticles electrodes fabrication by screen printing for wearable strain sensors. Surf. Coat. Technol. 2020, 384, 125308. [CrossRef]

9. Qaiser, N.; AlModaf, F.; Khan, S.M.; Shaikh, S.F.; ElAtab, N.; Hussain, M.M. A Robust Wearable Point-of-Care CNT-Based Strain Sensor for Wirelessly Monitoring Throat-Related Illnesses. Adv. Funct. Mater. 2021, 31, 2103375. [CrossRef]

10. Yamada, T.; Hayamizu, Y.; Yamamoto, Y.; Yomogida, Y.; Izadi-Najafabadi, A.; Futaba, D.N.; Hata, K. A stretchable carbon nanotube strain sensor for human-motion detection. Nat. Nanotechnol. 2011, 6, 296-301. [CrossRef]

11. Guo, X.; Huang, Y.; Zhao, Y.; Mao, L.; Gao, L.; Pan, W.; Zhang, Y.; Liu, P. Highly stretchable strain sensor based on SWCNTs/CB synergistic conductive network for wearable human-activity monitoring and recognition. Smart Mater. Struct. 2017, $26,095017$. [CrossRef]

12. He, Y.; Wu, D.; Zhou, M.; Zheng, Y.; Wang, T.; Lu, C.; Zhang, L.; Liu, H.; Liu, C. Wearable Strain Sensors Based on a Porous Polydimethylsiloxane Hybrid with Carbon Nanotubes and Graphene. ACS Appl. Mater. Interfaces 2021, 13, 15572-15583. [CrossRef] [PubMed]

13. Gong, S.; Lai, D.T.; Wang, Y.; Yap, L.W.; Si, K.J.; Shi, Q.; Jason, N.N.; Sridhar, T.; Uddin, H.; Cheng, W. Tattoolike Polyaniline Microparticle-Doped Gold Nanowire Patches as Highly Durable Wearable Sensors. ACS Appl. Mater. Interfaces 2015, 7 , 19700-19708. [CrossRef] [PubMed]

14. Amjadi, M.; Kyung, K.U.; Park, I.; Sitti, M. Stretchable, Skin-Mountable, and Wearable Strain Sensors and Their Potential Applications: A Review. Adv. Funct. Mater. 2016, 26, 1678-1698. [CrossRef]

15. Souri, H.; Banerjee, H.; Jusufi, A.; Radacsi, N.; Stokes, A.A.; Park, I.; Sitti, M.; Amjadi, M. Wearable and Stretchable Strain Sensors: Materials, Sensing Mechanisms, and Applications. Adv. Intell. Syst. 2020, 2, 2000039. [CrossRef]

16. Gao, Y.; Fang, X.; Tan, J.; Lu, T.; Pan, L.; Xuan, F. Highly sensitive strain sensors based on fragmentized carbon nanotube/polydimethylsiloxane composites. Nanotechnology 2018, 29, 235501. [CrossRef] [PubMed]

17. Li, T.; Li, J.; Zhong, A.; Han, F.; Sun, R.; Wong, C.P.; Niu, F.; Zhang, G.; Jin, Y. A flexible strain sensor based on CNTs/PDMS microspheres for human motion detection. Sens. Actuator A Phys. 2020, 306, 111959. [CrossRef]

18. Yang, Y.; Luo, C.; Jia, J.; Sun, Y.; Fu, Q.; Pan, C. A Wrinkled Ag/CNTs-PDMS Composite Film for a High-Performance Flexible Sensor and Its Applications in Human-Body Single Monitoring. Nanomaterials 2019, 9, 850. [CrossRef]

19. Shu, J.; Yang, R.; Chang, Y.; Guo, X.; Yang, X. A flexible metal thin film strain sensor with micro/nano structure for large deformation and high sensitivity strain measurement. J. Alloys Compd. 2021, 879, 160466. [CrossRef]

20. Amjadi, M.; Yoon, Y.J.; Park, I. Ultra-stretchable and skin-mountable strain sensors using carbon nanotubes-Ecoflex nanocomposites. Nanotechnology 2015, 26, 375501. [CrossRef] [PubMed]

21. Shi, G.; Zhao, Z.; Pai, J.H.; Lee, I.; Zhang, L.; Stevenson, C.; Ishara, K.; Zhang, R.; Zhu, H.; Ma, J. Highly Sensitive, Wearable, Durable Strain Sensors and Stretchable Conductors Using Graphene/Silicon Rubber Composites. Adv. Funct. Mater. 2016, 26, 7614-7625. [CrossRef]

22. Kahn, N.; Lavie, O.; Paz, M.; Segev, Y.; Haick, H. Dynamic Nanoparticle-Based Flexible Sensors: Diagnosis of Ovarian Carcinoma from Exhaled Breath. Nano Lett. 2015, 15, 7023-7028. [CrossRef] [PubMed]

23. Zheng, M.; Li, W.; Xu, M.; Xu, N.; Chen, P.; Han, M.; Xie, B. Strain sensors based on chromium nanoparticle arrays. Nanoscale 2014, 6, 3930-3933. [CrossRef] [PubMed]

24. Duan, S.; Wang, Z.; Zhang, L.; Liu, J.; Li, C. A Highly Stretchable, Sensitive, and Transparent Strain Sensor Based on Binary Hybrid Network Consisting of Hierarchical Multiscale Metal Nanowires. Adv. Mater. Technol. 2018, 3, 1800020. [CrossRef]

25. Ho, M.D.; Ling, Y.; Yap, L.W.; Wang, Y.; Dong, D.; Zhao, Y.; Cheng, W. Percolating Network of Ultrathin Gold Nanowires and Silver Nanowires toward "Invisible" Wearable Sensors for Detecting Emotional Expression and Apexcardiogram. Adv. Funct. Mater. 2017, 27, 1700845. [CrossRef]

26. Tang, W.; Yan, T.; Wang, F.; Yang, J.; Wu, J.; Wang, J.; Yue, T.; Li, Z. Rapid fabrication of wearable carbon nanotube/graphite strain sensor for real-time monitoring of plant growth. Carbon 2019, 147, 295-302. [CrossRef]

27. Lee, J.; Lim, M.; Yoon, J.; Kim, M.S.; Choi, B.; Kim, D.M.; Kim, D.H.; Park, I.; Choi, S.J. Transparent, Flexible Strain Sensor Based on a Solution-Processed Carbon Nanotube Network. ACS Appl. Mater. Interfaces 2017, 9, 26279-26285. [CrossRef] [PubMed]

28. Ma, L.; Yang, W.; Wang, Y.; Chen, H.; Xing, Y.; Wang, J. Multi-dimensional strain sensor based on carbon nanotube film with aligned conductive networks. Compos. Sci. Technol. 2018, 165, 190-197. [CrossRef]

29. Liu, H.; Gao, H.; Hu, G. Highly sensitive natural rubber/pristine graphene strain sensor prepared by a simple method. Compos. Part B Eng. 2019, 171, 138-145. [CrossRef] 
30. Yang, Y.F.; Tao, L.Q.; Pang, Y.; Tian, H.; Ju, Z.Y.; Wu, X.M.; Yang, Y.; Ren, T.L. An ultrasensitive strain sensor with a wide strain range based on graphene armour scales. Nanoscale 2018, 10, 11524-11530. [CrossRef]

31. Zhang, S.; Zhang, H.; Yao, G.; Liao, F.; Gao, M.; Huang, Z.; Li, K.; Lin, Y. Highly stretchable, sensitive, and flexible strain sensors based on silver nanoparticles/carbon nanotubes composites. J. Alloys Compd. 2015, 652, 48-54. [CrossRef]

32. Amjadi, M.; Pichitpajongkit, A.; Lee, S.; Park, I. Highly stretchable and sensitive strain sensor based on silver nanowire-elastomer nanocomposite. ACS Nano 2014, 8, 5154-5163. [CrossRef] [PubMed]

33. Liu, H.; Li, Q.; Zhang, S.; Yin, R.; Liu, X.; He, Y.; Dai, K.; Shan, C.; Guo, J.; Liu, C.; et al. Electrically conductive polymer composites for smart flexible strain sensors: A critical review. J. Mater. Chem. C 2018, 6, 12121-12141. [CrossRef]

34. Saetia, K.; Schnorr, J.M.; Mannarino, M.M.; Kim, S.Y.; Rutledge, G.C.; Swager, T.M.; Hammond, P.T. Spray-Layer-by-Layer Carbon Nanotube/Electrospun Fiber Electrodes for Flexible Chemiresistive Sensor Applications. Adv. Funct. Mater. 2014, 24, 492-502. [CrossRef]

35. Bai, J.B.; Allaoui, A. Effect of the length and the aggregate size of MWNTs on the improvement efficiency of the mechanical and electrical properties of nanocomposites-experimental investigation. Compos. Part A Appl. Sci. Manuf. 2003, 34, 689-694. [CrossRef]

36. Zhou, Y.; Zhan, P.; Ren, M.; Zheng, G.; Dai, K.; Mi, L.; Liu, C.; Shen, C. Significant Stretchability Enhancement of a Crack-Based Strain Sensor Combined with High Sensitivity and Superior Durability for Motion Monitoring. ACS Appl. Mater. Interfaces 2019, 11, 7405-7414. [CrossRef]

37. Chen, J.; Zhu, G.; Wang, F.; Xu, Y.; Wang, C.; Zhu, Y.; Jiang, W. Design of flexible strain sensor with both ultralow detection limit and wide sensing range via the multiple sensing mechanisms. Compos. Sci. Technol. 2021, 213, 108932. [CrossRef]

38. Liu, H.; Zhang, H.; Han, W.; Lin, H.; Li, R.; Zhu, J.; Huang, W. 3D Printed Flexible Strain Sensors: From Printing to Devices and Signals. Adv. Mater. 2021, 33, e2004782. [CrossRef] [PubMed]

39. Christ, J.F.; Aliheidari, N.; Potschke, P.; Ameli, A. Bidirectional and Stretchable Piezoresistive Sensors Enabled by Multimaterial 3D Printing of Carbon Nanotube/Thermoplastic Polyurethane Nanocomposites. Polymers 2018, 11, 11. [CrossRef] [PubMed]

40. Wajahat, M.; Lee, S.; Kim, J.H.; Chang, W.S.; Pyo, J.; Cho, S.H.; Seol, S.K. Flexible Strain Sensors Fabricated by Meniscus-Guided Printing of Carbon Nanotube-Polymer Composites. ACS Appl. Mater. Interfaces 2018, 10, 19999-20005. [CrossRef] [PubMed]

41. Zhu, X.; Xu, Q.; Li, H.; Liu, M.; Li, Z.; Yang, K.; Zhao, J.; Qian, L.; Peng, Z.; Zhang, G.; et al. Fabrication of High-Performance Silver Mesh for Transparent Glass Heaters via Electric-Field-Driven Microscale 3D Printing and UV-Assisted Microtransfer. Adv. Mater. 2019, 31, e1902479. [CrossRef] [PubMed]

42. Zhu, X.; Liu, M.; Qi, X.; Li, H.; Zhang, Y.F.; Li, Z.; Peng, Z.; Yang, J.; Qian, L.; Xu, Q.; et al. Templateless, Plating-Free Fabrication of Flexible Transparent Electrodes with Embedded Silver Mesh by Electric-Field-Driven Microscale 3D Printing and Hybrid Hot Embossing. Adv. Mater. 2021, 33, e2007772. [CrossRef]

43. Li, H.; Zhu, X.; Li, Z.; Yang, J.; Lan, H. Preparation of Nano Silver Paste and Applications in Transparent Electrodes via Electric-Field Driven Micro-Scale 3D Printing. Nanomaterials 2020, 10, 107. [CrossRef] [PubMed]

44. Zhang, H.; Qi, T.; Zhu, X.; Zhou, L.; Li, Z.; Zhang, Y.F.; Yang, W.; Yang, J.; Peng, Z.; Zhang, G.; et al. 3D Printing of a PDMS Cylindrical Microlens Array with 100\% Fill-Factor. ACS Appl. Mater. Interfaces 2021, 13, 36295-36306. [CrossRef]

45. Zhang, Y.F.; Li, Z.; Li, H.; Li, H.; Xiong, Y.; Zhu, X.; Lan, H.; Ge, Q. Fractal-Based Stretchable Circuits via Electric-Field-Driven Microscale 3D Printing for Localized Heating of Shape Memory Polymers in 4D Printing. ACS Appl. Mater. Interfaces 2021, 13, 41414-41423. [CrossRef] [PubMed]

46. Zhu, X.; Li, Z.; Hu, Y.; Li, H.; Yang, J.; Lan, H. Facile fabrication of defogging microlens arrays using electric field-driven jet printing. Opt. Laser Technol. 2020, 123, 105943. [CrossRef]

47. Chen, X.; Zeng, Q.; Shao, J.; Li, S.; Li, X.; Tian, H.; Liu, G.; Nie, B.; Luo, Y. Channel-Crack-Designed Suspended Sensing Membrane as a Fully Flexible Vibration Sensor with High Sensitivity and Dynamic Range. ACS Appl. Mater. Interfaces 2021, 13, 34637-34647. [CrossRef] [PubMed]

48. Nie, B.; Li, X.; Shao, J.; Li, X.; Tian, H.; Wang, D.; Zhang, Q.; Lu, B. Flexible and Transparent Strain Sensors with Embedded Multiwalled Carbon Nanotubes Meshes. ACS Appl. Mater. Interfaces 2017, 9, 40681-40689. [CrossRef] [PubMed] 